\title{
A PESCA ARTESANAL NO ALTO E MÉDIO RIO ARAGUARI , AMAPÁ, BRASIL
}

\author{
N. I. S. OLIVEIRA ${ }^{1,{ }^{*}}$, F. C. CUNHA ${ }^{2}$, L. PRESTES ${ }^{3}$, E. A. SANTOS ${ }^{4}$, M. G. M. SOARES ${ }^{5}$, A. C. FLORENTINO ${ }^{6}$
}

${ }^{1}$ Centro Integrado de Formação Profissional em Pesca e Aquicultura do Amapá, ${ }^{2,5}$ Universidade Federal do Amazonas, ${ }^{3,4}$ Universidade do Estado do Amapá, ${ }^{6}$ Universidade Federal do Amapá netieoliveira@gmail.com*

Submetido 03/01/2018 - Aceito 23/12/2018

DOI: $10.15628 /$ holos. 2018.6820

\section{RESUMO}

O presente estudo teve como objetivo avaliar a atividade pesqueira no alto e médio rio Araguari, Estado do Amapá. Aplicou-se 20 questionários aos pescadores associados à Colônia Z-16, com a finalidade de identificar os apetrechos de pesca, principais espécies capturadas, locais de pesca da atividade pesqueira no período de cheia e seca. A captura por unidade de esforço (CPUE) foi estimada através de dados de 1695 mapas de pesca do ano de 2003 a 2010. As embarcações foram caracterizadas por batelão e canoa, os principais petrechos descritos pelos pescadores foram o trapo/trapão, tiradeira, a rede de emalhar, bóia, linha de mão, caniço, trapinho, zagaia e tarrafa. As principais espécies explotadas são $\circ H$. aimara T.trilobatus, $A$. inermis $M$. asterias - Myloplus sp, $H$. malabaricus, $S$. rhombeus, Cichla, Pimelodus maculatus, L. trifasciatus, C. inornata, Prosomyleus rhomboidalis, Triportheus brachipomus e C. inornata. Os ambientes de pesca foram caracterizados por remansos, varjão, baixão, poços, corredeiras e igarapés. Os maiores picos de CPUE foi no período da seca, no mês de outubro de $2005 \mathrm{com}$ $105 \mathrm{~kg}$ pescador ${ }^{-1} \mathrm{dia}^{-1}$. A atividade é caracterizada como pesca artesanal comercial de pequena escala praticada por pescadores interioranos, multiespecífica e multiapetrecho.

PALAVRAS-CHAVE: atividade pesqueira, pescador, CPUE, Porto Grande.

\section{ARTISANAL FISHING IN THE HIGH AND MEDIUM ARAGUARI RIVER, AMAPÁ, BRAZIL}

\section{ABSTRACT}

The objective of this study was to evaluate the fishing activity in the middle Araguari River, State of Amapá. A total of 20 questionnaires were used to the fishermen associated with Colônia Z-16, in order to identify the fishing gear, main species caught, fishing sites of the fishing season during the flood and dry season, and estimated catch per unit of effort (CPUE) through data from 1695 fishing maps of the year 2003 to 2010. The vessels were characterized by barge and canoe, the main petrechos described by the fishermen were the rag / trap, gillnet, gillnet, float, hand line, caniço, trapinho, zagaia and tarrafa. The main exploited species are $\mathrm{H}$. aimara T. trilobatus, A. inermis M. asterias - Myloplus sp, H. malabaricus, S. rhombeus, Cichla, Pimelodus maculatus, L. trifasciatus, C. inornata, Prosomyleus rhomboidalis, Triportheus brachipomus and $\mathrm{C}$. inornata. The fishing environments were characterized by backwaters, varjão, baixão, wells, rapids and igarapés. The highest peaks of CPUE were in the dry season, in October 2005 with $105 \mathrm{~kg} /$ fisher $^{-1}$ day. The activity is characterized as small-scale commercial artisanal fishing practiced by inland fishermen, multispecies and multispecies.

KEYWORDS: Fishing activity, fisherman, cpue, Porto Grande. 


\section{INTRODUÇÃO}

A pesca artesanal é a atividade exercida por produtores autônomos, proprietários dos meios de produção, que exercem a pesca como atividade econômica prioritária, desenvolvendo relações de trabalho com base na parceria, considerada uma atividade secular, que prioritariamente utiliza-se mão de obra familiar, utilizando-se de apetrechos rudimentares à tecnologia de captura seletiva e não mecanizada, atuando em águas interiores, costeiras e estuarinas, que muita vezes concentra-se na captura de espécies mais rentáveis comercialmente, elevando a diminuição dos estoques pesqueiros (BARTHEM e FABRÉ, 2004; CLAUZET et al., 2005; MENDONÇA, 2015).

Em águas interiores, a pesca ocorre em lagos, igarapés e rios como exemplo temos o rio Araguari, que nasce nas montanhas do Tumucumaque e drena a porção central do Estado do Amapá cortando diversas Áreas Protegidas, como o Parque Nacional do Tumucumaque, Floresta Nacional do Amapá - FLONA e a Floresta Estadual do Amapá - FLOTA. Nessas áreas ocorre a atividade pesqueira que abastece os municípios de Porto Grande, Pedra Branca e localidades próximas como assentamentos e ribeirinhos que moram ao longo do curso d'água (BRANDÃO e SILVA, 2008; SOARES et al., 2012; CUNHA, 2017). Nos limites da FLONA com a FLOTA este rio encontra-se com um dos seus principais afluentes, o rio Falsino. No rio Falsino é proibida a atividade pesqueira profissional, enquanto que nos demais trechos do rio Araguari ela é permitida para todos os praticantes desde a sede do município de Porto Grande até a foz desse tributário. A partir deste ponto, a pesca só é permitida para afiliados da Colônia Z-16 apenas no rio Araguari e nos afluentes de sua margem direita, até a confluência com o rio Mutum (MOREIRA et al., 2011), divisa com o Parque Nacional Montanhas do Tumucumaque.

Estudos nestas áreas foram realizados como o de GAMA (2008) com levantamento de espécies e taxonomia na FLONA do Amapá e Parque Tumucumaque, BRANDÃO e SILVA (2008) acerca da atividade pesqueira em área da Unidade de conservação de uso sustentável; SOARES et al. (2012) com etnoconhecimento dos pescadores e dinâmica de população dos estoques pesqueiros; OLIVEIRA et al. (2013) investigando e avaliando os estoques pesqueiros, SANTOS et al. (2016) acerca do etnoconhecimento da alimentação dos pacus no alto e médio rio Araguari e CUNHA (2017) que abordou a conservação socioambiental da pesca em unidades de conservação utilizando a etnoecologia. Porém, a descrição de como ocorre a atividade pesqueira nestas áreas ainda são escassas, e, são de suma importância pois estas áreas estão sobre influência direta da hidrelétrica UHE Cachoeira Caldeirão (ECOTUMUCUMAQUE, 2011, 2013).

Neste contexto, este estudo caracterizou a atividade pesqueira no alto e médio rio Araguari exercida pelos pescadores do Município de Porto Grande, estado do Amapá possibilitando o entendimento de como esta atividade é praticada antes da implantação hidrelétrica e poderá ser utilizada em discussão de futura gestão da atividade pesqueira. Procuramos entender os tipos de pescarias praticadas, locais e espécies peixes capturados e a dinâmica da explotação do estoque pesqueiro nestas áreas. 


\section{METODOLOGIA}

\section{1 Área de estudo}

A bacia do rio Araguari apresentada na Figura 1 é a maior e mais importante bacia do Estado do Amapá, nasce na Serra do Tumucumaque e possui $617 \mathrm{~km}$ de extensão até a sua foz, no Oceano Atlântico (CUNHA, 2004). Conceitualmente é dividido em alto Araguari (132 km), médio Araguari (161 km), e baixo Araguari (205 km) (CUNHA, 2009).

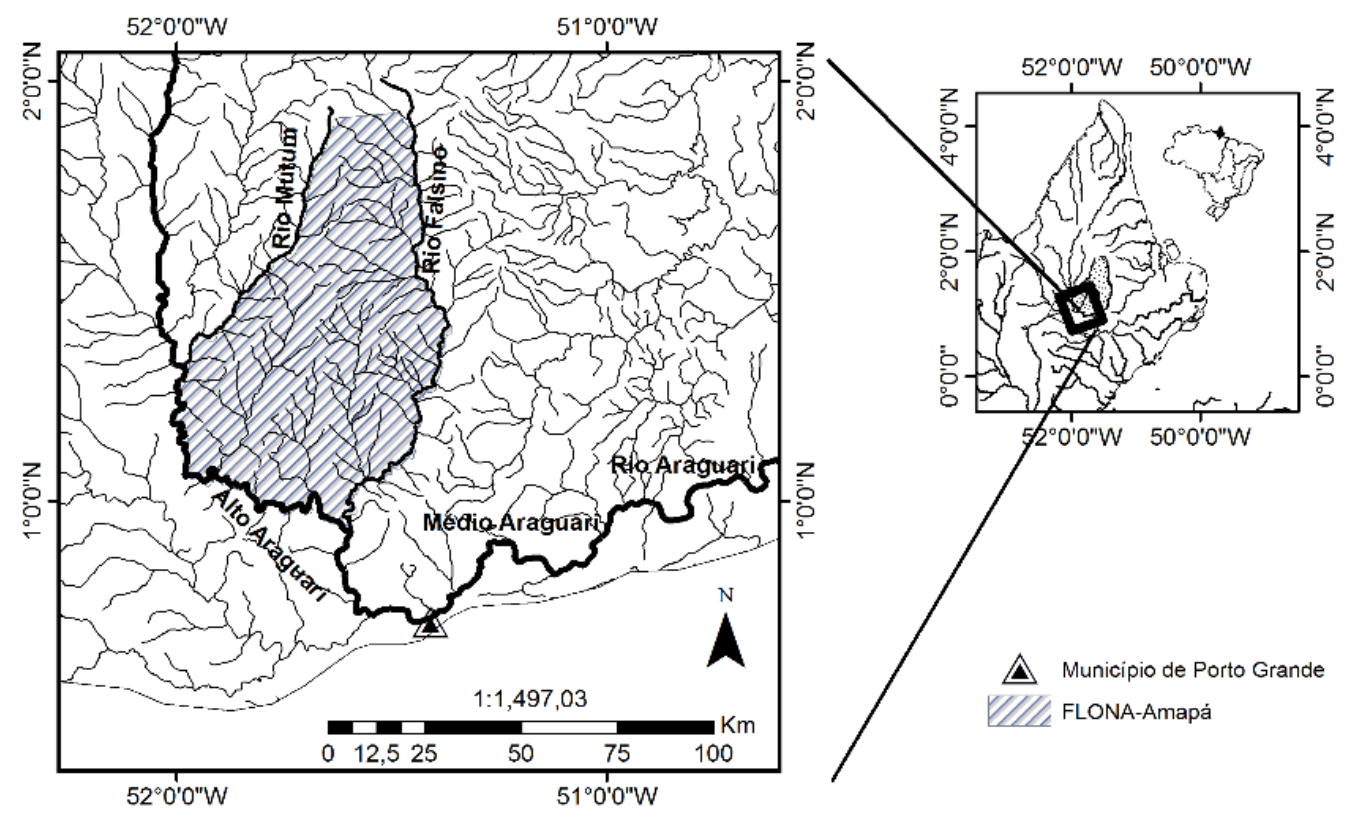

Figura 1. Localização do rio Araguari área de atividade pesqueira e município de Porto Grande onde está localizada a Colônia Z-16, Estado do Amapá, AP, BR.

\subsection{Amostragens}

As entrevistas foram realizadas entre os meses de fevereiro a agosto de 2012, que correspondem ao início das águas altas e baixas, respectivamente. Durante as entrevistas foram aplicados vinte (20) formulários com questionamentos padronizados possibilitando a comparação dos resultados (GIL, 2008), isso corresponde a aproximadamente $10 \%$ dos pescadores cadastrados na Colônia (RUFFINO, 2014). Os 646 mapas de pesca analisados foram confeccionados pelos próprios pescadores da Colônia Z-16 do ano de 2003 (17 mapas), 2005 (17 mapas), 2006 (56 mapas), 2007 (95 mapas), 2008 (132 mapas), 2009 (59 mapas) e 2010 (270 mapas) como forma de comprovar a pesca e receber o seguro defeso.

\subsection{Análise de dados}

A estatística descritiva e gráficos foram utilizadas para explorar os resultados. A Captura por Unidade de Esforço (CPUEp) foi utilizada para avaliar o esforço de pesca a partir das 
informações obtidas através de mapas de pesca da colônia de pescadores atuantes no alto e médio rio Araguari, essa análise foi realizada a partir da equação:

CPUEp $=\frac{P(\text { peso })}{\text { Esforço de pesca }}$

Onde: $\mathrm{CPUE}_{\mathrm{p}}=$ Captura por unidade de esforço em peso $(\mathrm{kg}) ; \mathrm{P}=$ Quantidade capturada de pescado em peso $(\mathrm{kg})$ e Esforço de pesca $=$ número de pescadores $\mathrm{X}$ dias de pescarias.

\section{RESULTADOS}

\subsection{Características atividade pesqueira}

Os resultados demonstram que 30\% $(n=6)$ dos pescadores da Colônia Z 16 exercem a atividade por aproximadamente 7 anos, 15\% ( $n=3), 6$ anos, 15\% $(n=3) 20$ anos, 10\% ( $n=2)$ por quatro anos, $5 \%(n=1), 5$ anos, $5 \%(n=1), 8$ anos, $5 \%(n=1), 10$ anos, $5 \%(n=1), 12$ anos, $5 \%(n=1), 13$ anos e $5 \%(n=1)$ exerce a atividade à 40 anos.

Apesar da pesca ser uma atividade tradicional e alguns declararem já praticarem há mais de vinte (20) anos, a maioria dos entrevistados neste estudo diz estar há menos de dez (10) anos na atividade pesqueira. OLIVEIRA et al. (2013) cita que o setor pesqueiro ligado ao município de Porto Grande era rentável para quem vivia exclusivamente da pesca, porém, com o aumento da quantidade de pessoas advindas de atividades que se tornaram ilegais (garimpo, extração madeireira etc.), o setor encontra-se inchado. Neste caso acredita-se que muitas pessoas estão adotando a atividade pesqueira nos últimos dez (10) anos como meio de sobrevivência (SOARES et al., 2012; CUNHA, 2017).

As embarcações de pesca utilizadas pelos pescadores variaram de 5 a 7 metros, destas $32 \%$ $(n=6)$ eram de 7 metros, 26\% $(n=5)$ de 8 metros, 15,8\% $(n=3)$ de 6 metros, 10,5\% $(n=2)$ de 10 metros, $10,5 \%(n=2) 7,5$ metros e $5,3 \%(n=1)$ de 5 metros. As embarcações motorizadas em sua maioria com potência de até 5,6 HP tem capacidade para suportar até 1,2 toneladas (conservados em isopor). Já às não motorizadas são de pequeno porte, com capacidade de até 0,5 tonelada, assoalho de madeira, movido a remo, com tripulação de 1 a 4 pescadores, é utilizada para navegação em locais mais secos como, por exemplo, em igarapés.

A frota que explora os recursos pesqueiros no alto e médio rio Araguari está constituída principalmente por embarcações de madeira considerando o universo amostral deste estudo que abrande $10 \%$ dos pescadores atuantes na Colônia Z-16. Um diagnóstico sobre o setor pesqueiro nesta área realizado por SOARES et al. (2012) descreveu 2 tipos de embarcações principais: batelão e canoa. Os batelões medem entre 6,5 a 7,2 metros, e utilizam diferentes tipos de motor, com potência de 3,5 a 5,6 HP, segundo RODRIGUES e TORRES (2007), trata-se de um motor que dá movimento e impulsiona as embarcações, sendo muito utilizada nos rios da Amazônia. Já as canoas variaram de 3 a 3,5 metros de comprimento e não possuem de propulsão motorizada. 
Os principais petrechos de pesca citados pelos pescadores foram o trapo/trapão $(95 \%, n=$ 19), a rede de emalhar (malha 50,60,70, 100, $120 \mathrm{~mm})(85 \%, \mathrm{n}=17)$, bóia $(60 \%, \mathrm{n}=12)$, caniço $(55 \%, n=11)$, linha de mão/anzol $(40 \%, n=8)$, trapinho $(45 \%, n=9)$, zagaia $(15 \%, n=3)$, tiradeira/ espinhel $(5 \%, n=1)$ e tarrafa $(5 \%, n=1)$, descritos no Quadro 1.

Quadro 1. Apetrechos utilizados pelos pescadores da Colônia Z-16 do município de Porto Grande, Amapá, AP.

\begin{tabular}{|c|c|c|}
\hline Apetrechos & Descrição & Autores \\
\hline Trapo/trapão & $\begin{array}{l}\text { Corda de nylon } \\
\text { trançado com anzol no. } 3 \text { a } \\
5 \text { usado com galhos de } \\
\text { árvores e enfiados no } \\
\text { substrato das margens do } \\
\text { rio para captura } \\
\text { principalmente do trairão }\end{array}$ & $\begin{array}{l}\text { SOARES et al. (2012); } \\
\text { SANTOS et al.(2016); } \\
\text { CUNHA (2017). }\end{array}$ \\
\hline Tiradeira/ espinhel & $\begin{array}{l}\text { Linha de nylon longa } \\
\text { com vários anzóis fixados } \\
\text { em linhas na vertical. Esses } \\
\text { anzóis geralmente são } \\
\left.\text { grandes (ex. } n^{\circ} 5\right)\end{array}$ & $\begin{array}{c}\text { CPESUL/IBAMA, } \\
2000, \text { COSTA et al.(2001) }\end{array}$ \\
\hline $\begin{array}{c}\text { Rede de emalhar } \\
\text { (malhadeira) }\end{array}$ & $\begin{array}{l}\text { Rede com variação } \\
\text { entre } 50,60,70,100,120 \\
\text { mm dos tamanhos entre nós } \\
\text { opostos, pode ser feita com } \\
\text { nylon com mono ou } \\
\text { multifilamento, possui bóias } \\
\text { na parte superior e } \\
\text { chumbadas na parte } \\
\text { inferior. }\end{array}$ & $\begin{array}{l}\text { HAMLEY (1975), REIS } \\
\text { e PAWSON (1992), } \\
\text { FERNANDES et al.(2009), } \\
\text { BATISTA et al. (2004). }\end{array}$ \\
\hline Bóia & $\begin{array}{l}\text { Linha de nylon com } \\
\text { anzol no. } 6 \text { a } 8 \text { usado em } \\
\text { garrafas plásticas flutuantes } \\
\text { e jogados nas corredeiras } \\
\text { para captura principalmente } \\
\text { dos pacus) }\end{array}$ & $\begin{array}{l}\text { SOARES et al. (2012); } \\
\text { SANTOS et al.(2016); } \\
\text { CUNHA (2017). }\end{array}$ \\
\hline Linha de mão/anzol & $\begin{array}{l}\text { As linhas utilizadas } \\
\text { podem ser de } 0,50 \mathrm{~mm} \text { e } \\
0,60 \mathrm{~mm} \text {. Normalmente se }\end{array}$ & $\begin{array}{l}\text { COSTA et al.(2001); } \\
\text { SOARES et al. (2012) }\end{array}$ \\
\hline
\end{tabular}




\begin{tabular}{|c|c|c|}
\hline & utiliza chumbada. & \\
\hline Trapinho & $\begin{array}{l}\text { Linha de nylon com } \\
\text { anzol no. } 6 \text { a } 8 \text { usado em } \\
\text { galhos de árvores no } \\
\text { varjão/baixão para captura } \\
\text { principalmente dos pacus) }\end{array}$ & $\begin{array}{l}\text { SOARES et al. (2012); } \\
\text { SANTOS et al.(2016); } \\
\text { CUNHA (2017). }\end{array}$ \\
\hline Caniço & $\begin{array}{l}\text { Anzol com linha e } \\
\text { caniço retirado da mata }\end{array}$ & $\begin{array}{l}\text { COSTA et al.(2001); } \\
\text { DORIA et al. (2012); SOARES } \\
\text { et al. (2012). }\end{array}$ \\
\hline Zagaia & $\begin{array}{l}\text { Com } \\
\text { aproximadamente } 2 \text { a } 2,5 \mathrm{~m} \\
\text { de comprimento e com } \\
\text { tridente fixado numa das } \\
\text { extremidades. }\end{array}$ & $\begin{array}{l}\text { ISAAC et al.(1996); } \\
\text { DANTAS (2011). }\end{array}$ \\
\hline Tarrafa & $\begin{array}{l}\text { Rede de pesca } \\
\text { circular feita com nylon } \\
\text { mono ou multifilamento } \\
\text { com pequenos pesos } \\
\text { distribuídos em torno de } \\
\text { toda a circunferência da } \\
\text { malha. }\end{array}$ & $\begin{array}{rc}\text { GAMBA, et } & \text { al. } \\
\text { (1994); GAMBA, (1994). } & \end{array}$ \\
\hline
\end{tabular}

Estudos realizados na Amazônia destacam a variedade de petrechos de pesca que são utilizados pelos pescadores para capturar de forma eficiente os peixes em variadas condições ambientais, sendo descritos mais de dez (10) tipos diferentes (PETRERE 1978; SMITH 1979; BARTHEM, 1999; BATISTA et al., 2004). Neste estudo, foram citados nove (9) apetrechos de pesca pelos entrevistados que utilizam a área da FLONA do Amapá para pescarias. Os apetrechos de pesca identificados expressam o uso de tecnologias tradicionais (como exemplo trapão/trapo, estiradeira, linha de mão) e modernas (malhadeira) (FURTADO, 1993). SOARES et al. (2012), destaca que a utilização do trapão é voltada principalmente para capturar o trairão e é utilizado das 18:00 horas até antes do amanhecer e a sua tendência de utilização é maior no verão.

A ocorrência contínua da utilização da rede de emalhar (verão e inverno) é observada neste estudo. A utilização da rede de emalhar nas embarcações não deve ser interpretada como preferência, visto que os proprietários destas usualmente utilizam outros petrechos, porém, esses relatam que utilizam a rede de emalhar para garantir a pescaria, mesmo das espécies que não possuem um expressivo retorno econômico. Enquanto, o trapão/trapo e trapinho são petrechos mais seletivos, que capturam espécies de porte maior e com um melhor retorno econômico, por exemplo, o curupeté ( $T$. trilobatus), madubé ( $A$. inermis) e trairão (H. aimara), não obstante estão 
cada vez mais numerosos ao longo do rio e, tem se capturado cada vez menos peixes e de tamanho menores segundo relato dos pescadores.

A frequência na utilização da rede de emalhar em toda a Amazônia é intensificada por permitir o manuseio por apenas uma pessoa, e possibilita o pescador atuar em outras atividades enquanto a rede está armada (PARENTE e BATISTA, 2005; BATISTA et al.,1998; CARDOSO et al., 2004; FREITAS e RIVAS, 2006 e FERNANDES et al., 2009), sendo descrita seu uso contínuo em outras áreas da Amazônia por FERNANDES et al. (2009) em Manaus e Manacapuru, DORIA et al. (2012) na bacia do rio Madeira-RO, BATISTA e ISAAC (2012) no Alto e Baixo Amazonas e no Alto Solimões, INOMATA e FREITAS(2015) no médio rio Negro e GARCEZ et al. (2017) no Lago Grande região no município de Manacapuru-AM. Em outras áreas no Brasil por RAMIRES e BARRELLA (2003) em Peruíbe-SP; CLAUZET e BARRELLA (2004) em Ubatuba (SP); LAUZET et al. (2007) em Guaibim (BA); por MARUYAMA et al. (2009) no médio e baixo rio Tietê-SP; SHORK et al. (2012) na região do alto rio Uruguai, reservatório de Machadinho; e MENDONÇA (2015) no litoral Sul de São Paulo.

A utilização do trapinho é semelhante a descrita por SOARES et al. (2012) e ocorre durante o dia, principalmente para capturar pacus. Na região do Alto Rio Tiquié (AM) esse apetrecho é muito utilizado por indígenas e denominado de linha de espera (CABALZAR, 2005) e, PETRERE (1978) descreve apetrecho semelhante, denominado curumim no estado do Amazonas. Muitas vezes a zagaia é utilizada de forma complementar, auxiliando os pescadores a retirar peixes maiores como o trairão e mais resistentes as capturas (SOARES et al., 2012). Esse apetrecho é utilizado nas pescarias artesanais na Amazônia e é amplamente descrito (SMITH, 1979; PETRERE, 1978; BARTHEM,1999). Os demais apetrechos como a tiradeira ou estiradeira (espinhel), caniço, linha de mão e a bóia são característicos de pesca de águas interiores e alguns são utilizados com maior frequência no inverno, como é o caso do trapinho e da bóia, enquanto outros são utilizados no inverno e verão como é o caso da malhadeira e a tiradeira (SOARES et al.,2012). O uso dos petrechos está relacionado ao tamanho e ao tipo de espécies de peixes que se deseja capturar bem como o período do ciclo hidrológico (PETRERE JR. et al.,2004; SOARES et al., 2012), afirmação corroborada pelos resultados deste estudo.

\subsection{Espécies explotadas pela atividade pesqueira}

Foram citadas uma diversidade de espécies, no período da cheia e seca que são explotadas pelos pescadores (Quadro 2). As mais citadas foram: Na seca: trairão (95\%, $n=19)$, curupeté (70\%, $n=14)$, mandubé $(65 \%, n=13)$, pacu-mafurá $(60 \%, n=12)$ pacu-branco $(25 \%, n=5)$, traíra-gapó $(20 \%, n=4)$, piranha $(15 \%, n=3)$, fusaca $(10 \%, n=2)$, tucunaré $(10 \%, n=2)$ e branquinha $(5 \%, n=1)$. No período da cheia, predominaram o pacu-mafurá $(79 \%, n=16)$, piranha $(74 \%, n=15)$, curupeté $(68 \%, n=14)$, trairão $(53 \%, n=11)$, pacu cumaru $(47 \%, n=9)$, e mandubé $(32 \%, n=6)$, aracu cabeça-gorda $(16 \%, n=3)$, fusaca $(11 \%, n=2)$, uéua $(11 \%, n=1)$, traíra gapó $(11 \%, n=1)$ e tucunaré $(11 \%, n=1)$ expostos no Quadro 2. 
Quadro 2. Nome popular e científico das espécies exploradas na pesca artesanal no alto e médio rio Araguari, Amapá, Brasil.

\begin{tabular}{|c|c|c|}
\hline Nome vulgar & Família & $\begin{array}{l}\text { Identificação } \\
\text { taxonômica }\end{array}$ \\
\hline Pacu curupeté & Characidae & $\begin{array}{l}\text { Tometes trilobatus } \\
\text { (Valenciennes, 1850) }\end{array}$ \\
\hline Pacu cumarú & Characidae & $\begin{array}{l}\text { Prosomyleus rhomboidalis } \\
\text { (Cuvier, 1818) }\end{array}$ \\
\hline Pacu mafurá & Characidae & $\begin{array}{l}\text { Myloplus asterias (MÜLLER } \\
\quad \text { \& TROSCHEL, 1844) }\end{array}$ \\
\hline Pacu branco & Characidae & Myloplus sp \\
\hline Pacu ferro & Characidae & Myleus sp \\
\hline Mandubé & Auchenipteridae & $\begin{array}{l}\text { Ageneiosus inermis } \\
\quad \text { (LINNAEUS, 1766) }\end{array}$ \\
\hline Branquinha & Curimatidae & $\begin{array}{ll}\text { Curimata } & \text { inormata } \\
\text { (VARI, 1989) }\end{array}$ \\
\hline Piranha-preta & Characidae & $\begin{array}{l}\text { Serrasalmus rhombeus } \\
\quad \text { (LINNAEUS, 1766) }\end{array}$ \\
\hline Trairão & Erythrinidae & $\begin{array}{l}\text { Hoplias aimara } \\
\text { (VALENCIENNES, 1847) }\end{array}$ \\
\hline Traira gapó & Erythrinidae & $\begin{array}{l}\text { Hoplias malabaricus } \\
\quad(\mathrm{BLOCH}, 1794)\end{array}$ \\
\hline Aracú-cabeça-gorda & Anostomidae & $\begin{array}{cc}\text { Leporinus } & \text { trifasciatus } \\
\text { (STEINDACHNER, 1876) }\end{array}$ \\
\hline Mandí-fusaca & Pimelodidae & $\begin{array}{l}\text { Pimelodus maculatus } \\
\text { (LACEPÈDE, 1803) }\end{array}$ \\
\hline Uéua com listra & Acestrorhynchidae & $\begin{array}{l}\text { Acestrorhynchys } \\
\text { falcirostris (CUVIER, } \\
\text { 1819) }\end{array}$ \\
\hline Uéua & Acestrorhynchidae & $\begin{array}{l}\text { Acestrorhynchys } \\
\text { falcatus (BLOCH, 1794) }\end{array}$ \\
\hline
\end{tabular}




\begin{tabular}{l|l|l}
\hline Tucunaré & Cichlidae & Cichla sp. \\
\hline Sarda & Characidae & $\begin{array}{c}\text { Triportheus brachipomus } \\
\text { (VALENCIENNES, 1850) }\end{array}$ \\
\hline
\end{tabular}

As espécies de peixes capturadas nas atividades de pesca artesanal na região é semelhante às citadas por BRANDÃO e SILVA (2008) ao fazer a descrição do conhecimento ecológico dos pescadores da Floresta Nacional do Amapá-FLONA; no relatório técnico de bioecologia para Floresta nacional do Amapá-FLONA-AP de SOARES et al. (2012), OLIVEIRA et al. (2013) ao avaliar os estoques pesqueiros no rio Araguari, SANTOS et al. (2016) ao fazer uma análise do etnoconhecimento dos pescadores sobre o regime alimentar dos pacus, CUNHA (2017) ao fazer a avaliação da conservação socioambiental da pesca em unidades de conservação utilizando a etnoecologia.

\subsection{Ambientes de Pesca}

No rio Araguari existe pontos de pesca pré-determinados pelos pescadores locais, com a ocorrência de habitats propícios a pesca, sendo identificados e descritos como remansos, varjão, baixão, poços, corredeiras e igarapés descritos no Quadro 3.

Quadro 3- Ambientes de pesca utilizados na pesca artesanal no alto e médio rio Araguari, Amapá, Brasil.

\begin{tabular}{|c|c|c|c|}
\hline $\begin{array}{l}\text { Ambiente de } \\
\text { Pesca* }\end{array}$ & Ocorrência & $\begin{array}{l}\text { Espécies } \\
\text { capturadas }\end{array}$ & $\begin{array}{l}\text { Apetrecho } \\
\mathrm{S}\end{array}$ \\
\hline Remansos & \begin{tabular}{l}
\multicolumn{2}{c}{ Zonas de } \\
menor correnteza \\
imediatamente \\
posteriores \\
corredeiras.
\end{tabular} & \begin{tabular}{l}
\multicolumn{1}{c}{ Myloplus, } \\
M.asterias, \\
T.trilobatus,Prosomyl \\
eus rhomboidalis.
\end{tabular} & $\begin{array}{l}\text { Tiradeira, } \\
\text { rede de emalhar, } \\
\text { caniço e bóia, }\end{array}$ \\
\hline Varjões & $\begin{array}{l}\text { Áreas alagadas } \\
\text { nas margens do rio, } \\
\text { onde árvores, } \\
\text { geralmente frutíferas, } \\
\text { estão adaptadas ao } \\
\text { maior período de } \\
\text { alagação. }\end{array}$ & 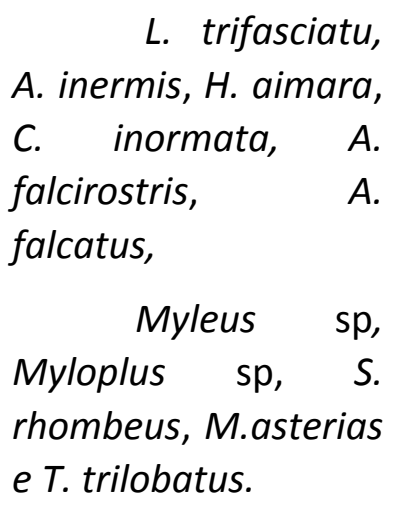 & $\begin{array}{l}\text { Trapinho e } \\
\text { o caniço. }\end{array}$ \\
\hline Baixões & Áreas alagadas & . trifasciatus, & Caniço, \\
\hline
\end{tabular}




\begin{tabular}{|c|c|c|c|}
\hline & $\begin{array}{l}\text { com presença de } \\
\text { árvores, área aberta e } \\
\text { baixa velocidade da } \\
\text { água, geralmente } \\
\text { localizadas na entrada } \\
\text { de igarapés. }\end{array}$ & $\begin{array}{l}\text { A. inermis, aimará, } \\
\text { M. asterias. }\end{array}$ & rede de emalhar \\
\hline Poços & $\begin{array}{l}\text { Zonas com } \\
\text { maior profundidade } \\
\text { do leito do rio, e, } \\
\text { menor correnteza. }\end{array}$ & \begin{tabular}{l}
\multicolumn{2}{c}{ A.inermis, } \\
H.aimara, \\
rhomboidalis \\
M.asterias
\end{tabular} & $\begin{array}{l}\text { Linha de } \\
\text { mão e rede de } \\
\text { emalhar }\end{array}$ \\
\hline Corredeiras & $\begin{array}{l}\text { Jusante da } \\
\text { cachoeira, em áreas } \\
\text { de remanso e baixão. }\end{array}$ & $\begin{array}{l}\text { T.trilobatus e } \\
\text { (S. rhombeus, Myleus } \\
\text { sp e Myloplus sp. }\end{array}$ & Bóias \\
\hline Igarapés & $\begin{array}{l}\text { Afluentes do } \\
\text { rio com leito estreito } \\
\text { e menor que dos rios, } \\
\text { a água geralmente são } \\
\text { claras e de } \\
\text { temperatura baixa } \\
\text { devido a maior } \\
\text { cobertura da floresta. }\end{array}$ & $\begin{array}{l}\text { H.aimara e } M \text {. } \\
\text { asterias. }\end{array}$ & $\begin{array}{l}\text { Rede de } \\
\text { emalhar, trapão e } \\
\text { trapo. }\end{array}$ \\
\hline
\end{tabular}

* Elaborado de acordo com as informações do estudo e baseado em SOARES et al. (2012); SANTOS et al. (2016) e CUNHA (2017).

Os pescadores artesanais da Colônia Z-16 utilizam como pontos de pesca principalmente o alto e médio rio Araguari, área onde está situada a Floresta Nacional do Amapá - FLONA, a Floresta Estadual do Amapá - FLOTA, unidades com fins de manejo, e a divisa do Parque Montanhas do Tumucumaque (IBAMA, 2007), área de preservação. Os habitats mais utilizados nas pescarias foram o remanso, varjão e baixão, que ocorrem principalmente no interior da FLONA (alto rio Araguari), que apresenta alta abundância das espécies de peixe explotadas comercialmente. As utilizações desses ambientes de pesca são citados por BRANDÃO (2008), SOARES et al. (2012), SANTOS et al. (2016) e CUNHA (2017).

Vale ressaltar que o EIA/RIMA e o Plano Básico Ambiental - PBA (ECOTUMUCUMAQUE, 2011,2013) descrevem um impacto hidrológico da construção UHE da Cachoeira Caldeirão sobre o alto e médio rio Araguari como certo, não significativo, permanente e irreversível, afirmam ainda que a capacidade de mitigação é baixa, pois depende da adequada gestão da bacia hidráulica visando a redução do aporte de sedimentos. Esses estudos (EIA/RIMA e PBA) indicaram que o alcance da formação do lago da UHE seria até a Foz do rio Amapari, confluência com rio Araguari, nesse sentido, é possível concluir que os ambientes de pesca no alto e médio rio Araguari não 
serão afetados (vazão, ciclo de inundação, disponibilidades de ambientes) pela construção desta hidrelétrica.

\subsection{Quantificação do total do pescado desembarcado (CPUE)}

Foram analisados 646 mapas de pesca do ano de 2003 a 2010. A quantidade total de pescado capturado registrada nos mapas de pesca foi de $39.034 \mathrm{Kg}$ de peixes. Para a captura por unidade de esforço a quantidade de pescadores que atuaram em 2003 foi de 13 pescadores, capturando um total de $1.125 \mathrm{Kg}$ de peixes, e nesse ano o mês que apresentou maior captura foi outubro. No ano de 2004, não foram localizados e/ou não foram feitos registros. Já no ano de 2005, 13 pescadores capturaram $1.653 \mathrm{Kg}$, e o mês que apresentou a maior captura foi setembro. No ano de 2006, 26 pescadores capturaram $4.624 \mathrm{Kg}$, o mês que apresentou maior captura foi maio. No ano de 2007, 64 pescadores capturaram $6.379 \mathrm{Kg}$ e o mês com maior captura foi junho. Em 2008, 100 pescadores capturaram $7.686 \mathrm{Kg}$ com maior captura em abril. Em 2009, 41 pescadores capturaram $6.224 \mathrm{Kg}$, com maior pico de captura em abril. Finalmente em 2010, 203 pescadores capturaram $11.343 \mathrm{Kg}$ de peixes e junho teve maior valor total capturado (Tabela 2; Figura 2).

Considerando o esforço pesqueiro (CPUE) no desembarque, o ano de 2003 apresentou um total de $93,61 \mathrm{~kg} /$ pescador ${ }^{-1}$ dia e o mês com maior captura foi agosto. No ano de 2005 , o total capturado foi de $332,2 \mathrm{~kg} /$ pescador $^{-1} \mathrm{dia}$, e, o mês com maior captura foi outubro. No ano de 2006, o total foi de $59,81 \mathrm{~kg} /$ pescador $^{-1}$ dia, e, o mês de maior captura foi maio. No ano de 2007 , o total capturado foi $25,54 \mathrm{~kg} /$ pescador $^{-1} \mathrm{dia}$, já o mês com maior captura foi novembro. $\mathrm{O}$ ano de 2008 apresentou total de captura de $13,18 \mathrm{~kg} /$ pescador $^{-1}$ dia, e, o mês com maior captura foi novembro. Em 2009, o total de captura foi de $72,59 \mathrm{~kg} /$ pescador $^{-1}$ dia, e, o mês com maior captura foi novembro. No ano de 2010, o total capturado foi de $5,28 \mathrm{~kg} /$ pescador $^{-1}$ dia, e, nesse ano o mês que apresentou maior captura foi março, listados na Tabela 2 e Figura 2.

Tabela 2. Desembarque pesqueiro (sem e com esforço) gerado a partir de mapas de pesca confeccionados pelos pescadores do médio e alto rio Araguari. Fonte: Colônia de pesca Z-16, referentes aos anos de 2003, 2005, 2006, 2007, 2008, 2009 e 2010.

\begin{tabular}{llllllllll}
\hline & \multicolumn{7}{c}{ Quantidade de pescado sem esforço (kg) } \\
\cline { 2 - 9 } Anos & Março & Abril & Maio & Junho & Julho & Agosto & Setembro & Outubro & Novembro \\
\hline 2003 & 80 & 195 & 205 & 70 & 70 & 100 & 185 & 220 & - \\
2005 & 244 & 105 & 196 & 262 & 100 & 246 & 290 & 210 & - \\
2006 & 430 & 635 & 660 & 617 & 470 & 506 & 489 & 631 & 186 \\
2007 & 289 & 894 & 884 & 1015 & 864 & 695 & 909 & 773 & 56 \\
2008 & 529 & 1324 & 1020 & 868 & 986 & 985 & 836 & 704 & 434 \\
2009 & 756 & 899 & 885 & 815 & 853 & 760 & 861 & 265 & 130 \\
2010 & 789 & 1233 & 1773 & 2188 & 2032 & 786 & 655 & 1254 & 633 \\
\hline
\end{tabular}




\begin{tabular}{llllllllll} 
& \multicolumn{7}{c}{} & \multicolumn{7}{c}{ (CPUE no. pescador/dia) } \\
\cline { 2 - 8 } Anos & Março & Abril & Maio & Junho & Julho & Agosto & Setembro & Outubro & Novembro \\
\hline 2003 & 16 & 9 & 10 & 14 & 8,36 & 20 & 9,25 & 7 & - \\
2005 & 24,5 & 35 & 24,5 & 26,2 & 50 & 30,75 & 36,25 & 105 & - \\
2006 & 9,58 & 5,02 & 11,6 & 5,6 & 4,91 & 4,1 & 4,23 & 5,1 & 9,6 \\
2007 & 4,82 & 1,49 & 1,19 & 1,57 & 1,47 & 2,94 & 1,09 & 2,61 & 8,36 \\
2008 & 1,64 & 0,81 & 0,62 & 0,88 & 0,89 & 0,8 & 1,22 & 1,8 & 4,52 \\
2009 & 7,27 & 6,53 & 4,13 & 3,16 & 3,74 & 3,8 & 4,37 & 7,12 & 32,5 \\
2010 & 1,24 & 0,4 & 0,41 & 0,44 & 0,61 & 0,49 & 0,36 & 0,4 & 0,93 \\
\hline
\end{tabular}

Os dados de desembarque revelam que o período de cheia foi que apresentou os maiores picos, expostos na Figura 2A, entretanto, a CPUE revela que o maior rendimento foi no período da seca, verificados na Figura 2B.

A)

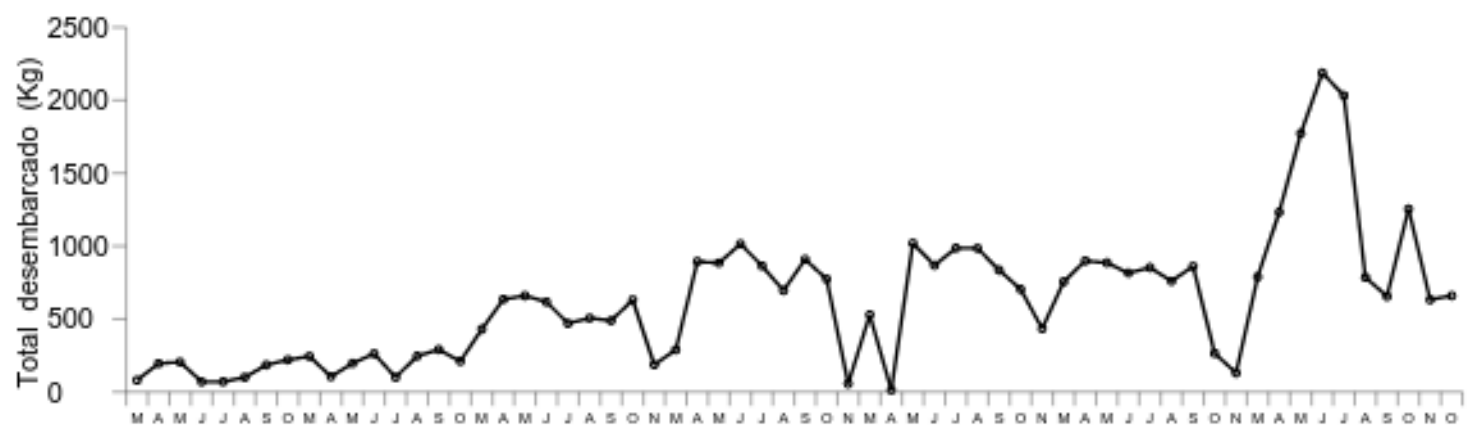

B)

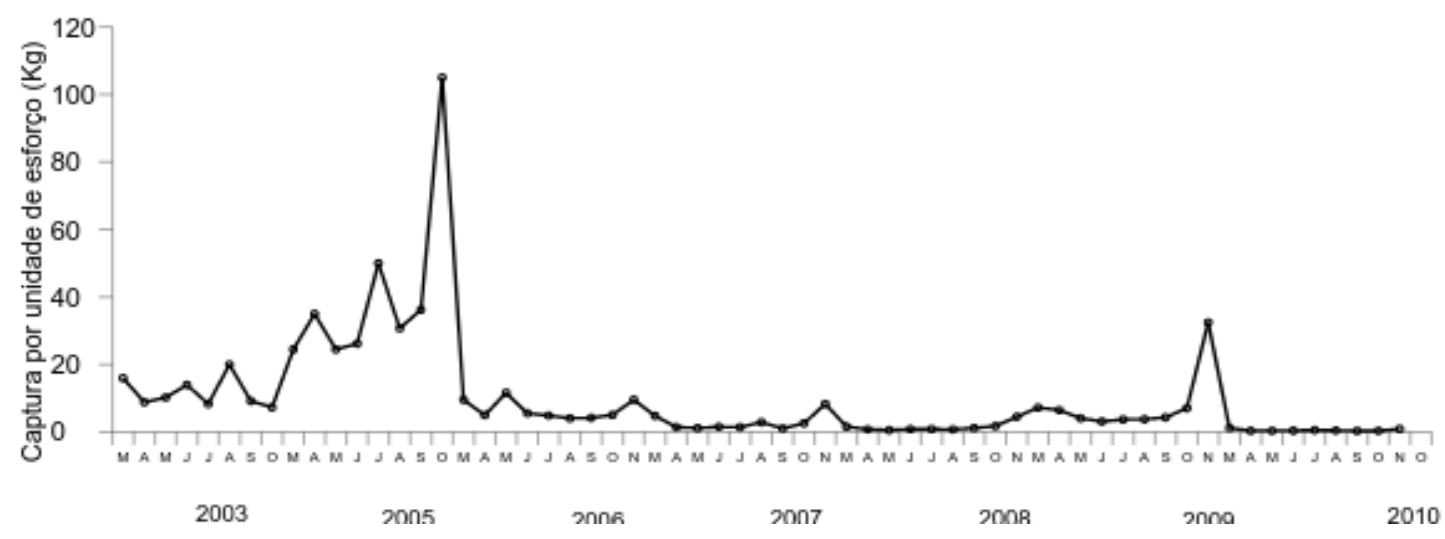

Figura 2. Desembarque pesqueiro dos anos de 2003 a 2010 da pesca artesanal no alto e médio rio Araguari, Amapá, Brasil. (A) dados brutos sem considerar o esforço pesqueiro e (B) Captura por Unidade de Esforço em Kg. 
O resultado desta pesquisa registrou maiores picos de CPUE no período da seca, no mês de outubro de 2005 com $105 \mathrm{~kg} /$ pescador $^{-1}$ dia, esse maior rendimento da CPUE no período da seca é descrito nos resultados de LIMA et al. (2016) no município de Humaitá, Amazonas onde os maiores rendimentos pesqueiros foram durante o período da seca, de 43,6 (pescador ${ }^{-1}$ dia) ${ }^{-1}$ no mês outubro de 2009. Umas das justificativas para que a captura seja maior no período da vazante e seca no rio Araguari é que nessa época a nível do rio diminui fazendo com que as espécies sejam expostas e vulneráveis nos abrigos que antes estavam com grande fluxo de água, este fato colabora para que elas sejam capturadas mais facilmente utilizando-se de apetrechos diversos. Isso ocorre em outras regiões (BATISTA e PETRERE JR, 2003; CARDOSO e FREITAS, 2007; GONÇALVES e BATISTA, 2008; DORIA et al., 2012). Vale ressaltar que os valores de desembarque mostram um aumento das capturas desde o ano de 2003 até o ano de 2010, porém, ao analisar os valores de CPUE (kg/pescador ${ }^{-1} \mathrm{dia}$ ) nos anos de 2003 a 2009 os valores estão razoáveis, porém no ano de 2010 apresentou uma mudança severa, onde a captura diminuiu muito por pescador devido principalmente ao aumento no número de pescadores e quantidade de pescarias. Neste período, a atividade pesqueira sofreu mudanças drásticas, como restrição de pontos de pesca, assim como o aumento considerável de pescadores na área do rio Araguari com a criação das unidades de conservação e preservação (IBAMA, 2007), o fechamento das atividades de mineração e proibição de retirada de madeira, que praticamente limitaram os pescadores a uma pequena área do alto e todo o médio rio Araguari. E, essas áreas disponíveis para pesca no rio Araguari não estão conseguindo comportar o drástico aumento dos pescadores que fazem parte da Associação Z-16, e os demais pescadores não associados.

\section{CONCLUSÃO}

Os resultados expostos neste trabalho descrevem como ocorre a atividade pesqueira evidenciando espécies capturadas, petrechos e embarcações utilizados para a captura desse pescado, ambientes de pesca e quantidade capturada $(\mathrm{kg})$ por ano no médio e alto rio Araguari, e, esta pode ser classificada como pesca artesanal comercial de pequena escala praticada por pescadores interioranos, multiespecífica e multiapetrecho.

Esta informação poderá ser utilizada para subsidiar medidas de manejo e permitindo a identificação de padrões na atividade pesqueira assim como possíveis impactos, exemplo são as construções hidroelétricas. Estudos realizados nestas áreas são importantes e poderão subsidiar acordos e leis restritas à área da pesca e uso de recursos pesqueiros para o avanço do processo de geração do conhecimento e integração do homem ao ambiente onde vive, garantindo que o aumento no esforço de pesca não dirija o estoque a depleção e a ineficiência econômica.

\section{REFERÊNCIAS}

BARTHEM R. B.; FABRÉ, N. N. (2004) Biologia e diversidade dos recursos pesqueiros na Amazônia. In: Ruffino, M.L. (Coord.) A pesca e os recursos pesqueiros na Amazônia brasileira Manaus Ibama /Próvárzea p. 17- 62. 
BARTHEM, R. B., PETRERE JR., M.; ISSAC, V.; RIBEIRO, M. C. L. D. B., MCGRATH, D. G., VIEIRA, I. J e BARCO, M. V. (1997) "A pesca na Amazônia: problemas e perspectivas para o seu manejo". Em Valladares-Pádua, C. e Bodmer, R. E. (eds.). Manejo e conservação de vida silvestre no Brasil. Rio de Janeiro, MCT/ CNPq/ Sociedade Civil Mamirauá, p 173-185.

BARTHEM, R.B. (1999) A Pesca Comercial no Médio Solimões e sua Interação com a Reserva de Desenvolvimento Sustentável Mamirauá. pp.72-107. In: Queiroz, H.; Cramp, L. \&Willliam, G. R. (orgs.). Estratégias para Manejo de Recursos Pesqueiros em Mamirauá. Sociedade Civil Mamirauá/CNPq, Belém.

BATISTA, V.S. (1998) Distribuição, dinâmica da pesca e dos recursos pesqueiros na Amazônia Central. 291f. Tese de doutorado, INPA/FUA; Manaus. Disponível em: http://koha.inpa.gov.br/cgi-bin/koha/opac

detail.pl?biblionumber=13103\&shelfbrowse_itemnumber=19388. Acesso: 29 Set 2017.

BATISTA, V.S.; Petrere Jr., M. (2003) Characterization of the commercial fish production landed at Manaus. Acta Amazonica, 33(2): 291-302.

BATISTA, V. S.; ISSAC, V. J.; VIANA, J. P. (2004) Exploração e manejo dos recursos pesqueiros da Amazônia. In: A Pesca e os Recursos Pesqueiros na Amazônia Brasileira. Ruffino, M. L. (Coord.) - Manaus: IBAMA/ProVárzea, p. 63 - 151.

BATISTA, V.S.; ISAAC, V.J. (2012) Peixes e pesca no Solimões-Amazonas: uma avaliação integrada. 1 a ed. Brasília: IBAMA. 278p.

BRANDÃO, F. C.; SILVA L. M. (2008) Conhecimento ecológico tradicional dos pescadores da Floresta Nacional do Amapá. Uakari, 4(2):55-66.

CABALZAR, A (Org.). (2005) Peixe e Gente no Alto rio Tiquié: conhecimentos tukano e tuyuka, ictiologia, etnologia. São Paulo: Instituto Sócio Ambiental, 339p.

CARDOSO, R. S.; BATISTA, V.S.; JÚNIOR, C. H. F.; MARTINS, W.R. (2004) Aspectos econômicos e operacionais das viagens da frota pesqueira de Manaus, Amazônia Central. Acta Amazonica, 34(2): 301-307.

CARDOSO, R.S.; FREITAS, C.E.C. (2007) Desembarque e esforço de pesca da frota pesqueira comercial de Manicoré (Médio Rio Madeira), Amazonas, Brasil. Acta Amazonica, 37(4): 605612.

CLAUZeT, M. e BARRELLA, W. (2004) A pesca artesanal na Praia Grande do Bonete, Ubatuba, Litoral Norte de São Paulo. In: DIEGUES, A.C. Enciclopédia Caiçara. Núcleo de Apoio a Pesquisa sobre Populações Humanas em Áreas Úmidas Brasileiras - NUPAUB. 1:147- 161. 
CLAUZET, M.; RAMIRES, M.; BARRELLA, W.A. (2005) Pesca artesanal e conhecimento local de duas populações caiçaras (enseada do mar virado e barra do una) no litoral de São Paulo, Brasil. Multiciência: A Linguagem da Ciência, 4, 1-22.

CEPSUL/IBAMA,.(2000) Informe da pesca extrativa marinha em Santa Catarina 1998. Itajaí. p. 36.

COSTA, L. R. F da.; BARTHEM,, R.B.; BITTENCOURT, M. M.; (2001) A pesca do tambaqui, Colossoma macropomum, com enfoque na área do Médio Solimões. Acta Amazonica, 31(3):449-468.

CUNHA, A.C. Dossiê sobre a Implementação do Centro de Monitoramento e Previsão de Tempo, Clima e Recursos Hídricos do Estado do Amapá. (2004) Macapá, AP: IEPA/LABHIDRO. 91p.

CUNHA, A.C. (2009) Uso do sistema de modelagem Qual2e para o estudo de impacto ambiental na qualidade da água causado pela barragem e cidades ribeirinhas no Alto e Médio rio AraguariAmapá. Relatório Final de Projeto. NHMET/IEPA. Processo CNPq (Edital Universal), No. 479405, 2006-9.147.

CUNHA, F.C. (2017) Etnoecologia da pesca: um caminho para a conservação socioambiental da pesca em unidades de conservação. 180f. (Tese de doutorado em Ciências Pesqueiras nos Trópicos. Programa de Pós-Graduação em Ciências nos Trópicos-Manaus).

DANTAS, M. S.S. (2011) A vida comanda o rio: etnoecologia de pescadores de três comunidades do rio Cuieiras, Baixo rio Negro, Amazonas, 92f. (Dissertação de Mestrado. Instituto Nacional de Pesquisas da Amazônia). Disponível em: <http://bdtd.inpa.gov.br/handle/tede/778> Acesso em: 18 out. 2017.

DORIA, C.R.C.; RUFFINO, M.L.; HIJAZI, N.C.; CRUZ, R.L. (2012) A Pesca comercial na bacia do rio Madeira, estado de Rondônia, Brasil. Acta Amazonica, 42(1): 29-40.

ECOTUMUCUMAQUE. (2011) Estudo de impacto ambiental e relatório de impacto ambiental (EIARIMA) da Hidrelétrica Cachoeira Caldeirão. Amapá.

ECOTUMUCUMAQUE. (2013) Plano Básico Ambiental do Aproveitamento Hidrelétrico Cachoeira Caldeirão. Volume III - Programa meio socioeconômico. Amapá.

FERNANDES V.L.A.; VICENTINI R.F.; BATISTA, V.S.(2009) Caracterização do uso de malhadeiras pela frota pesqueira que desembarca em Manaus e Manacapuru, Amazonas. Acta Amazonica, 39(2): 405-414.

FREITAS, C.E.C.; RIVAS, A.A.F. (2006) A pesca e os recursos pesqueiros na Amazônia Ocidental. Ciência e Cultura (SBPC), 58 (3): 30-32.

FURTADO, L. G. (1993) Pescadores do rio Amazonas: um estudo antropológico da pesca ribeirinha numa área amazônica. Belém: Museu Paraense Emilio Goeldi. $486 f$. 
GAMA, C. de S. (2008) A criação de tilápia no estado do Amapá como fonte de risco ambiental. Acta Amazônica, 38(3): 525 - 530.

GAMBA, M. da R. (1994) Guia prático de tecnologia de pesca. - Itajaí: IBAMA/CEPSUL: Santa Catarina, 94p il.

GAMBA, M. da R.; BAILON M. A., CONOLL Y P. C.(1994) Catálogo das artes de pesca artesanal do estado de Santa Catarina. Itajaí: IBAMA/CEPSUL, Santa Catarina, 119 p. il.

GARCEZ, R. C. S.; SOUZA, L. A. de; FRUTUOSO, Márcia Elane; FREITAS, Carlos Edwar de Carvalho. (2017) Seasonal dynamic of Amazonian small-scale fisheries is dictated by the hydrologic pulse. Boletim Instituto de Pesca, 43(2): $207-221$.

GIL, Antonio Carlos. (2008) Como elaborar projetos de pesquisa. 4. ed. São Paulo: Atlas. 176p.

GONÇALVES, C.; BATISTA, V.S. (2008) Avaliação do desembarque pesqueiro efetuado em Manacapuru, Amazônia Central. Acta Amazonica, 38(1): 135-144.

HAMLEY, J.M. (1975) Review of gill-net selectivity. Journal of the Research Board of Canada 32: 1943-1969.

INOMATA, S.O.; FREITAS, C.E.C. (2015) A pesca comercial no médio rio Negro: aspectos econômicos e estrutura operacional. Boletim do Instituto de Pesca, 41(1): 79-87.

INSTITUTO BRASILEIRO DO MEIO AMBIENTE DOS RECURSOS NATURAIS RENOVAVEIS- IBAMA. (2007) Estatística da pesca Brasil/Grandes regiões e unidades da federação. Diretoria de Uso Sustentável da Biodiversidade e Florestas - DBFLO Coordenação Geral de Autorização de Uso e Gestão da Fauna e Recursos Pesqueiros CGFAP. Brasília. Disponível em: http://www.ibge.gov.br/censo2010/primeiros_dados_. Acesso em: 30 Ago 2017.

INSTITUTO BRASILEIRO DO MEIO AMBIENTE DOS RECURSOS NATURAIS RENOVAVEIS- IBAMA. (2000) Centro de Pesquisas e Extensão Pesqueira das Regiões Sudeste e Sul. 130. (Coleção Meio Ambiente). Série Estudos Pesca, 16.

ISAAC, V.J. ; MELSTEIN, A. ; RUFFINO, M.L. (1996) A pesca artesanal no Baixo Amazonas: análise multivariada da captura por espécie. Acta Amazônica, 26(3): 185-208.

LAUZET, M.; RAMIRES, M.; BEGOSSI, A. (2007) Etnoictiologia dos pescadores artesanais da Praia de Guaibim, Valença (BA), Brasil. Neotropical Biology and Conservation, 2(3): 136-154.

LIMA, M. A. L.; FREITAS , C. E. de C.; MORAES, S. M. de; DORIA, C. R. da C. (2016) Pesca artesanal no município de Humaitá, Médio Rio Madeira, Amazonas, Brasil. Boletim Instituto de Pesca, 42(4): 914-923. 
MARUYAMA, S., CASTRO, P.M.G., PAIVA, P. (2009) Pesca artesanal no Médio e Baixo Tietê, São Paulo, Brasil: Aspectos estruturais e socioeconômicos. Boletim Instituto de Pesca, São Paulo, 35(1): $61-81$.

MENDONÇA, J.T. (2015) Caracterização da pesca artesanal no litoral Sul de São Paulo -Brasil. Boletim Instituto de Pesca, 41(3): 479 - 492.

MOREIRA, H.L.F. (2011) Marudá: aspectos da mudança social em uma comunidade de pescadores da Amazônia: FURTADO, L.; MELLO, A:F: \& Povos das Águas realidade e perspectiva na Amazônia. LEITÃO, W. (eds.) . p. 119-132.

OLIVEIRA, N. I. DA S. DE. SOUZA, L. P. DE, FLORENTINO, A. C.; SOARES, M. G. M.; CAVALCANTE, B. R. S. (2013) Avaliação dos estoques pesqueiros explotados pela pesca artesanal no Médio e Alto Rio Araguari, Amapá, Brasil. Revista Ciência Da Amazônia. 3(2).

PARENTE, V.M.; BATISTA, V.S. (2005) A organização do desembarque e o comércio de pescado de 1990 em Manaus, Amazonas. Acta Amazonica. 35 (3): 375-382.

JR, M. (1978) Pesca e esforço de pesca no Estado do Amazonas- Locais, aparelhos de captura e estatísticas de desembarque. Acta Amazônica, 3(2): 1978, 54.

PETRERE JUNIOR, M. (2004) Setor pesqueiro: análise da situação atual e tendências do desenvolvimento da indústria da pesca. Manaus, Ibama/PróVarzea.

RAMIRES, M. e BARRELLA, W. (2003) Ecologia da pesca artesanal em população caiçara de Juréia Itatins, São Paulo, Brasil. Interciencia, 28(4): 208-213.

REIS, E.G.; PAWSON, M.G. (1992) Determination of gillnet selectivity for bass (Dicentrarchus labrax L.) using commercial catch data. Fisheries Research, 13(2): 173-187.

RODRIGUES, L.M.; TORRES, I.C. (2007) Relações de Gênero na Comunidade São Francisco de Paroá na costa do Canbuoca, Manacapuru, Am. In: FABRÉ, N.N; BATISTA, V. S.; WAICHMAN, A. V.. RIBEIRO, M.O. A.S. ; PRANG, G. Sociodiversidade e conservação da Várzea Amazônica (Ogs.). Editora Aran, Manaus, PYRÀ, p.83-88.

RUFINO, M. (2014) Diagnostico da pesca e aquicultura no estado do Amapá. Macapá. GSA/Conservação Internacional-Cl.

SANTOS, A. L. CUNHA, F. C. da; SOARES, M. G. M.; PRESTES, L.;FLORENTINO, A.C. (2016) Etnoconhecimento dos pescadores sobre o regime alimentar dos pacus (Serrasalminae) do Médio Rio Araguari, AP. Biotemas , 29(2): 101-111. 
SCHORK, G., HERMES-SILVA, S.,BEUX, L.F.,ZANIBONI-FILHO, E., NUÑER, A.P.O. (2012) Diagnóstico da pesca artesanal na Usina Hidrelétrica de Machadinho, Alto Rio Uruguai - Brasil .Boletim Instituto de Pesca, 38(2): 97 - 108.

SMITH, N.J.H. (1979) A pesca no rio Amazonas. Manaus: INPA, 154p.

SOARES, M.G.M., BATISTA G., CUNHA, F.C., PRESTES, L.P.S. (2012) Bioecologia e etnoecologia da ictiofauna na Floresta Nacional do Amapá como subsídio para o manejo do recurso pesqueiro. 